\title{
Efeito de EPDM Modificado nas Propriedades Mecânicas e no Processamento de Composições de EPDM Contendo Resíduo de EPDMR
}

\author{
Danielli M. Carmo, Vanessa de S. Francisco, Carolina G. F. Rezende, Paulo J. Oliveira \\ Departamento de Engenharia Química, UFRRJ
}

\author{
Ademir J. Zattera \\ Departamento de Engenharia Química, UCS \\ Márcia G. Oliveira \\ Divisão de Processamento e Caracterização de Materiais, INT
}

\begin{abstract}
Resumo: Neste trabalho foi estudado o efeito de três agentes na recuperação de resíduos de borracha, copolímero de etileno-propylenedieno (EPDMR) contendo negro de fumo. Os três agentes preparados foram: EPDM modificado com 1-dodecanotiol (EPDMSDD), EPDM modificado com ácido esteárico (EPDMAE) e EPDM modificado com ácido graxo linolênico (EPDMAG). Os resultados de propriedades mecânicas mostraram que o EPDMSDD fornece a melhor combinação de resistência à tração e rasgamento associado à processabilidade. A adição dos agentes EPDMAE e EPDMAG melhorou a processabilidade da mistura por extrusão, mantendo suas propriedades mecânicas. Este resultado indica que o EPDMAE e o EPDMAG atuam como auxiliares de processamento.
\end{abstract}

Palavras-chave: Borracha, reciclagem, resíduo, adesão.

\section{Effect of Modified EPDM on the Mechanical Properties and Processing of EPDM Containing EPDM Waste}

\begin{abstract}
This work was aimed at examining the effect from three agents in the recycling of waste rubber, namely copolymer ethylenepropylene-diene (EPDMR) containing carbon black. Three agents were prepared: EPDM modified by 1-dodecanotiol (EPDMSDD), EPDM modified by stearic acid (EPDMAE) and EPDM modified by fatty linolenic acid (EPDMAG). For the mechanical properties, the better tensile and tear strength associated with manufacturing extrusion were obtained using EPDMSDD. EPDMAE and EPDMAG improved the processing by extrusion, while keeping the mechanical properties. These results indicate that EPDMAE and EPDMAG act as auxiliary agents for processability.
\end{abstract}

Keywords: Rubber, recycling, waste, adhesion.

\section{Introdução}

Os artefatos de borrachas apresentam ampla aplicação tecnológica que se estende deste a indústria automobilística até as aplicações as hospitalares ${ }^{[1]}$.

Os polímeros, de um modo geral, apresentam degradação lenta e nas borrachas (elastômeros), este fato é potencializado pela presença de ligações cruzadas e de outros aditivos presentes na composição como antioxidantes, antiozonantes e cargas que dificultam não somente a degradação, como também o seu reaproveitamento, pois afetam o processamento da mistura ${ }^{[2]}$.

Uma forma de reutilização de resíduos de borracha é transformá-lo em pó, e utilizá-lo como carga em novas formulações. Este procedimento apresenta como vantagem o baixo custo, mas, de acordo com a literatura, apenas pequenas quantidades de carga, pós-uso, pode ser adicionada à borracha virgem sem causar perda de propriedades fisico-mecânicas e/ou afetar a processabilidade (viscosidade) da mistura ${ }^{[6]}$.

Einstein formalizou as primeiras equações que descrevem os efeitos da presença de partículas sólidas no escoamento de fluidos e na tensão de cisalhamento baseados na mecânica do contínuo. Essas equações serviram como base matemática na elaboração de modelos para descrever sistemas concentrados ${ }^{[3]}$.
PAYNE mostrou os principais efeitos da adição de cargas, como o negro de fumo e a sílica, nas propriedades reológicas. Suas pesquisas possibilitaram compreender a participação e o papel da distribuição e da dispersão de cargas na viscoelasticidade da mistura ${ }^{[4]}$. Mais recentemente, Fröhlich e colaboradores têm utilizado a reologia como ferramenta para compreender a interação polímero-carga e a formação de agregados através dos ensaios de viscoelasticidade ${ }^{[5]}$

Uma das maneiras de melhorar a adesão entre poliméricas tem sido o uso de polímeros modificados, copolímero em bloco ou graftizado, capaz de reduzir a tensão interfacial e melhorar a distribuição entre as fases ${ }^{[7]}$. Esses agentes se localizam preferencialmente na interface, polímero-polímero ou partículapolímero, melhorando a adesão $0^{[8]}$.

Baeta e colaboradores investigaram o uso de borracha SBR modificada com 1 dodecanotiol (SBRSDD) como agente compatibilizante na reciclagem de borracha SBR (SBR-r) em matriz de NBR. Os resultados mostraram que a presença do agente compatibilizante melhorou a processabilidade e as propriedades mecânicas das mistura, como também permitiu uma maior incorporação do resíduo SBR-r na mistura ${ }^{[9,10]}$.

Autor para correspondência: Paulo J. Oliveira, Departamento de Engenharia Química, Universidade Federal Rural do Rio de Janeiro - UFRJ, 
Nessa direção, este trabalho tem como objetivo estudar três tipos de agentes: EPDMSDD, EPDMAE e o EPDMAG no reaproveitamento de resíduos de borracha EPDM (EPDMR) investigando as propriedades mecânicas e o processamento das composições de EPDM.

\section{Experimental}

A Borracha EPDM (copolímero de etileno-propilenonorboneno) (EPDM 65), gentilmente cedida pela DSM Elastômeros do Brasil S.A,: massa molecular $(\mathrm{Mn})=150,000$, viscosidade de Mooney $\left(\mathrm{ML} 1+4\right.$ at $\left.100{ }^{\circ} \mathrm{C}\right)=77$, (60/40) razão de etilenopropileno e 2,5\% em massa de norboneno. O resíduo de borracha EPDM (vedação de janelas de ônibus) (EPDMR) foi recolhido como descarte na indústria automobilística. O EPDMR foi moído, separado e selecionado a seguinte faixa de diâmetro de partícula 0,252 a 0,358 mm. O ácido esteárico (AE), enxofre (S), óxido de zinco $(\mathrm{ZnO})$ e tolueno, Vetec química fina, grau de pureza comercial. 2,2-dissulfeto de mercaptobenzotiazila (MBTS) e Dissulfeto de tetrametiltiuram (TMTD), Indústrias Monsanto S.A., São Paulo, SP, grau de pureza comercial. 1-dodecanotiol 98\%, Research Quemicals Ltda, Alemanha.

\section{Preparação das misturas}

As misturas foram preparadas num misturador interno a $80{ }^{\circ} \mathrm{C}$, tempo total de processamento de 21 minutos, e ordem de adição

Tabela 1. Formulação da básica das misturas EPDM/EPDMR em phr (partes por 100 partes de borracha).

\begin{tabular}{cccccc}
\hline Ingredientes & \multicolumn{5}{c}{ Misturas } \\
\hline & MO & MR & SDD & AE & AG \\
\hline EPDM $^{\mathrm{a}}$ & 100 & 100 & 100 & 100 & 100 \\
EPDMR $^{\mathrm{b}}$ & - & 80 & 80 & 80 & 80 \\
EPDMSDD $^{\mathrm{c}}$ & - & - & 5 & - & - \\
EPDMAE $^{\mathrm{d}}$ & - & - & - & 5 & - \\
EPDMAG $^{\mathrm{e}}$ & - & - & - & - & 5 \\
ZnO $^{\mathrm{f}}$ & 5 & 5 & 5 & 5 & 5 \\
Enxofre $_{\text {Ár }}$ & 2 & 2 & 2 & 2 & 2 \\
Ácido Estárico $^{\mathrm{T}}$ & 1 & 1 & 1 & 1 & 1 \\
MMTD $^{\mathrm{g}}$ & 0,2 & 0,2 & 0,2 & 0,2 & 0,2 \\
\hline
\end{tabular}

${ }^{\mathrm{a} B o r r a c h a}$ EPDM (copolímero de etileno-propileno-norboneno),

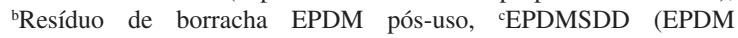
modificado com 1-dodecanotiol), ${ }^{\mathrm{d}}$ EPDMAE (EPDM modificado com ácido esteárico), ${ }^{\circ}$ EPDMAG (EPDM modificado com ácido graxo), ${ }^{\mathrm{f}}$ Óxido de Zinco, ${ }^{\mathrm{g}}$ Dissulfeto de tetrametiltiuram e ${ }^{\mathrm{h}} \mathrm{T}$ Tetrametiltiuram.

(a)

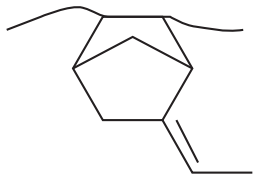

(b)

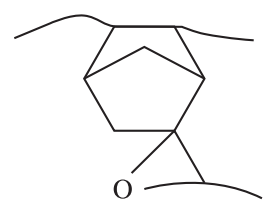

dos componentes conforme apresentado na Tabela 1. As misturas foram prensadas a 6,7 MPa, segundo norma ASTM D 3182-89, nos respectivos tempos de vulcanização (t90) para cada formulação.

\section{Preparação dos agentes}

A síntese dos agentes, bem como suas estruturas é apresentada na Figura 1.

\section{Sintese do EPDMSDD}

Vinte gramas de EPDM forma solubilizados em $300 \mathrm{~mL}$ de tolueno a $80{ }^{\circ} \mathrm{C}$, sob atmosfera de nitrogênio e agitação por 10 minutos. Em seguida, adicionou-se $10 \mathrm{~mL}$ de 1-dodecanotiol em quantidade molar com base na quantidade de norborneno presente no EPDM (2,5\%). Transcorrido o tempo de reação de 4 horas, a solução foi purificada com etanol e seca sob vácuo. O EPDMSDD foi caracterizado por Ressonância Magnética Nuclear de ${ }^{13} \mathrm{C}$.

\section{Síntese do EPDM modificado com grupo epóxido (EPDMEP)}

Para a síntese dos agentes EPDMAE e EPDMAG, o EPDM foi primeiramente epoxidado e posteriormente procedeu-se a síntese dos agentes. A metodologia utilizada para epoxidação da borracha EPDM foi a mesma utilizada na literatura ${ }^{[10-12]}$.

\section{Síntese dos agentes EPDMAE e EPDMAG}

O EPDMEP foi solubilizado em $500 \mathrm{~mL}$ tolueno (na proporção de $1 \mathrm{~g}$ de polímero/ $15 \mathrm{~mL}$ de tolueno), sob atmosfera de nitrogênio, a $75^{\circ} \mathrm{C}$ por 20 minutos. Para síntese do EPDMAE foram adicionados $10 \mathrm{~mL}$ ácido esteárico em tolueno, e para síntese do EPDMAG foram adicionados $10 \mathrm{~mL}$ ácido linoleico. Nas duas síntese foram utilizados $0,2 \mathrm{~mL}$ de $\mathrm{HCl}$ como catalisador. Ao termino da reação, 4 horas, os polímeros foram precipitados em acetona, lavados com água, secos. O material foi caracterizado por FTIR.

\section{Ensaios mecânicos}

Foram realizados ensaios de resistência à tração, deformação e resistência ao rasgamento das misturas apresentadas na Tabela 1, conforme descrito nas normas (ASTM D 412-98) e (ASTM D 62498), respectivamente.

\section{Processamento por extrusão}

As misturas foram processadas em extrusora na temperatura de $60{ }^{\circ} \mathrm{C}$ em todas as zonas de aquecimento, a $10 \mathrm{RPM}$, usando uma matriz tipo Garvey. Esta matriz tem a geometria definida no método ASTM 2230-96.

(c)

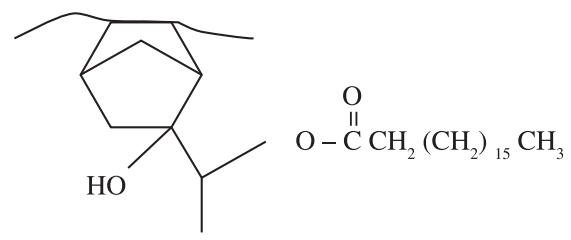

(e)

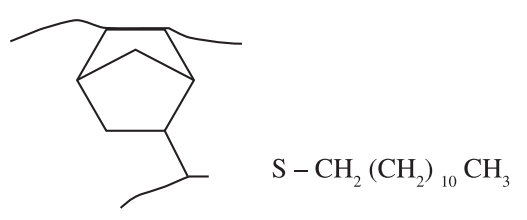

Figura 1. a) EPDM; b) EPDMEP; c) EPDMAE; d) EPDMAG; e e) EPDMSDD. 


\section{Resultados e Discussão}

\section{Caracterização dos agentes}

Oespectro de FTIR da borracha EPDMé apresentado na Figura 2. Notam-se algumas bandas de interesse: em $808,04 \mathrm{~cm}^{-1}$ referente à dupla ligação do norboneno, pois será na insaturação que ocorrerá a reação de epoxidação. Após a reação de epoxidação ocorreu o surgimento de uma banda em $871 \mathrm{~cm}^{-1}$ atribuída à deformação axial simétrica do anel epóxi, confirmando a reação de epoxidação. Wang e colaboradores relataram que durante a epoxidação do EPDM podem ocorrer reações secundárias com o grupamento epóxi dando origem a carbonila de éster, éter ou éter cíclico, cujas bandas são respectivamente: 17301178 e 1100-1000 cm-1[10]. Estas reações não foram observadas no EPDM epoxidado, Figura 2.

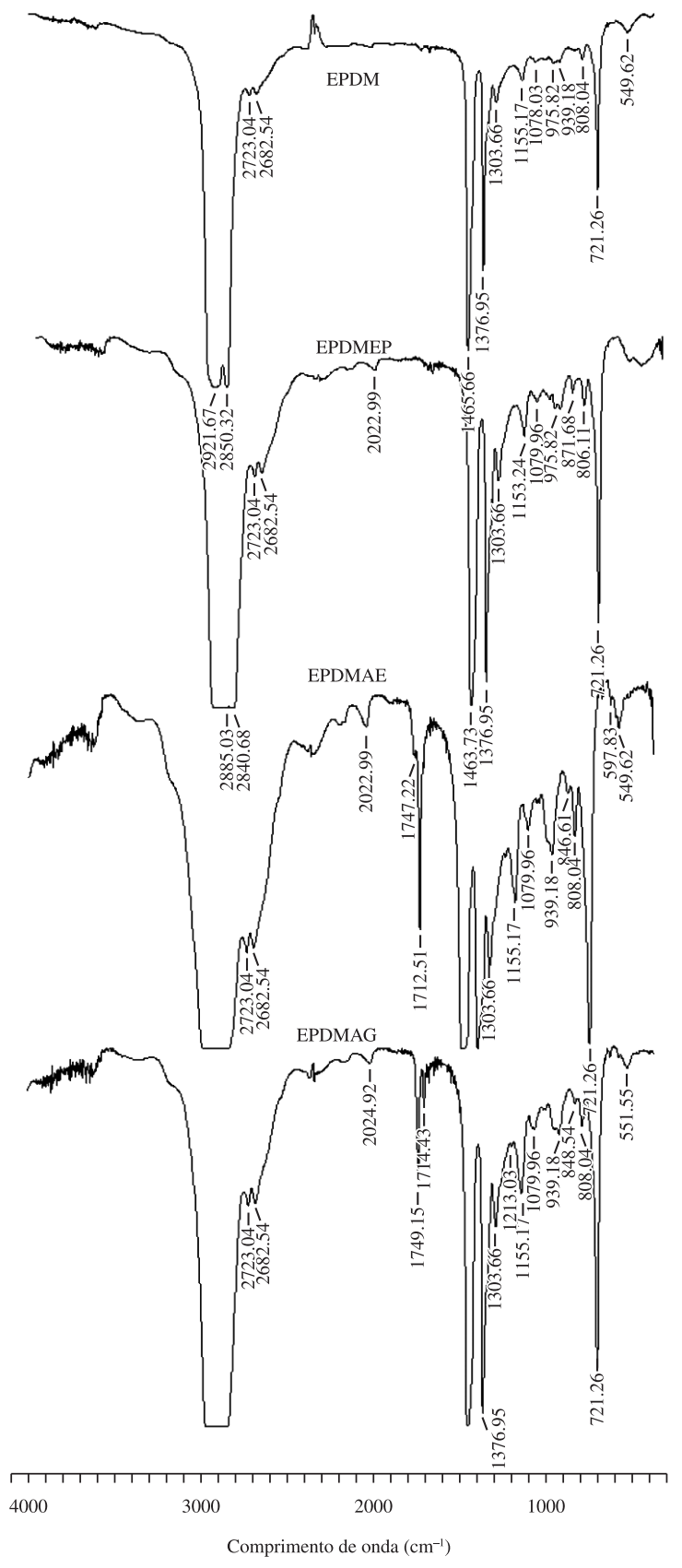

Figura 2. FTIR do EPDM, EPDMEP, EPDMAE e EPDMAG.
$\mathrm{O}$ grupo $\mathrm{C}=\mathrm{O}$ dos ácidos carboxílicos apresentam bandas na região de $1760 \mathrm{~cm}^{-1}$. Em se tratando dos ácidos alifáticos saturados, a deformação $\mathrm{C}=\mathrm{O}$ pode aparecer na região de $1720-1706 \mathrm{~cm}^{-1[13]}$. Na Figura 2 observa-se o desaparecimento da banda referente a epoxidação, $871 \mathrm{~cm}^{-1}$ e o aparecimento das bandas referentes a reação com ácido esteárico; 1712,51 e 1747,22 cm-1, e para o reação com ácido linoleico, 1714,43 e 1749,15 cm $\mathrm{cm}^{-1}$.

Considerando que o espetro de IR para o EPDMSDD é de difícil resolução, optou-se em caracterizá-lo por RMN. A Figura 3 mostra os espectros de 13C RNM do EPDM e do EPDMSDD. Os sinais atribuídos aos sulfetos situam-se entre 10 e 80 ppm $^{[13]}$. Comparando os espectros observa-se que os sinais 21,45; 22,69; 33,11 e 34,42 ppm, presentes do EPDMSDD, não estão presentes no espectro de ${ }^{13} \mathrm{C}$ NMR do EPDM. Carmem e colaboradores estudaram a presença de óleo extensor e de polipropileno na mistura EPDM/ $\mathrm{PP}$, comparando com um espectro de polipropileno isotático ${ }^{[14-16]}$. Para o espectro de 13C NMR do EPDM/PP, os autores observaram sinais em 22,8 e 32,1 ppm, os quais foram atribuídos às respostas da cadeia butílica terminal do óleo extensor, pois os sinais referentes ao óleo não estão presentes no espectro do EPDM puro. Podemos atribuir os sinais encontrados em 21,45, 22, 69, 33,11 e 34,42 ppm a presença do cadeia do dodecanotiol funcionalizada no EPDM.

$\mathrm{Na}$ Figura $4 \mathrm{a}$ verifica-se que a mistura sem carga (MO) é apresenta menor valor de resistência à tração, comparada com a mistura (MR). Este resultado está associado ao efeito do EPDMR como carga de reforço, pois contém negro de fumo.

Verifica-se que a presença dos agentes EPDMSDD e EPDMAE aumenta a resistência à tração quando comparada com a amostra MR, há um aumento de 15 e 7,5\% respectivamente. Entretanto, o agente EPDMAG pouco afetou a resistência à tração, aumento de $2,5 \%$, próximo ao desvio padrão da amostra. Este resultado sugere que o EPDMSDD e EPDMAG conferem uma maior adesão entre as fases EPDMR/EPDM.

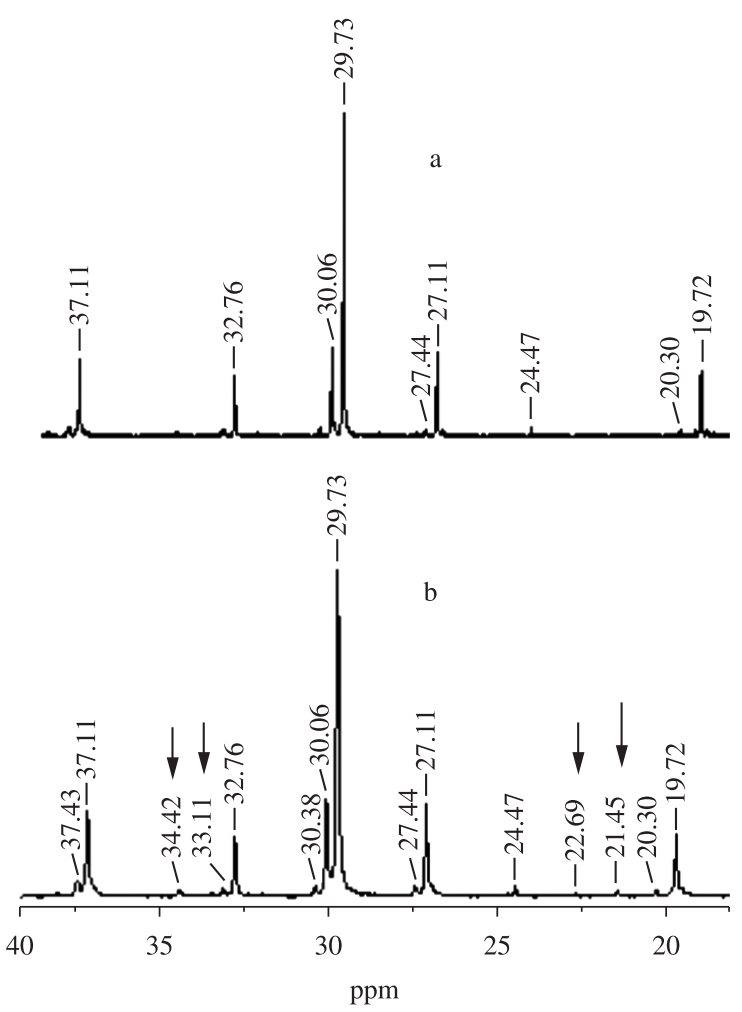

Figura 3. Espectroscopia de Ressonância Magnética Nuclear de 13C RMN: a) EPDM; e b) EPDMSDD. 


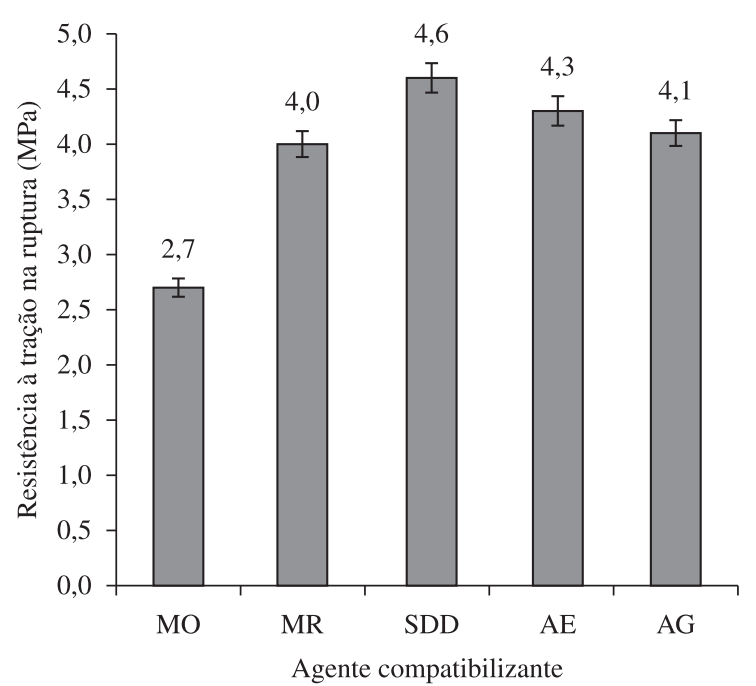

(a)

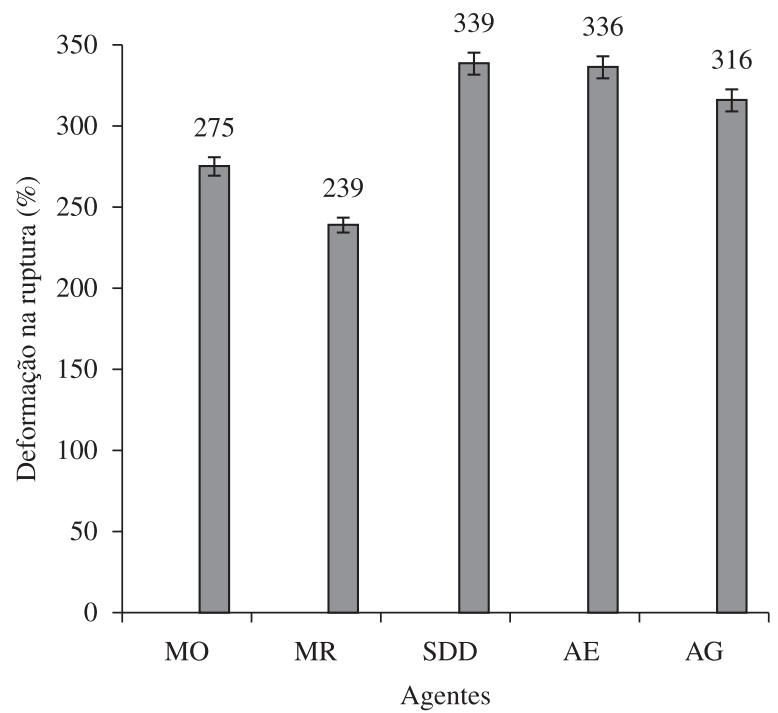

(b)

Figura 4. Efeito do teor de agente na resistência à tensão na ruptura: a) margem de erro de $\pm 3 \%$ e alongamento na ruptura; e b) margem de erro de $\pm 5 \%$.

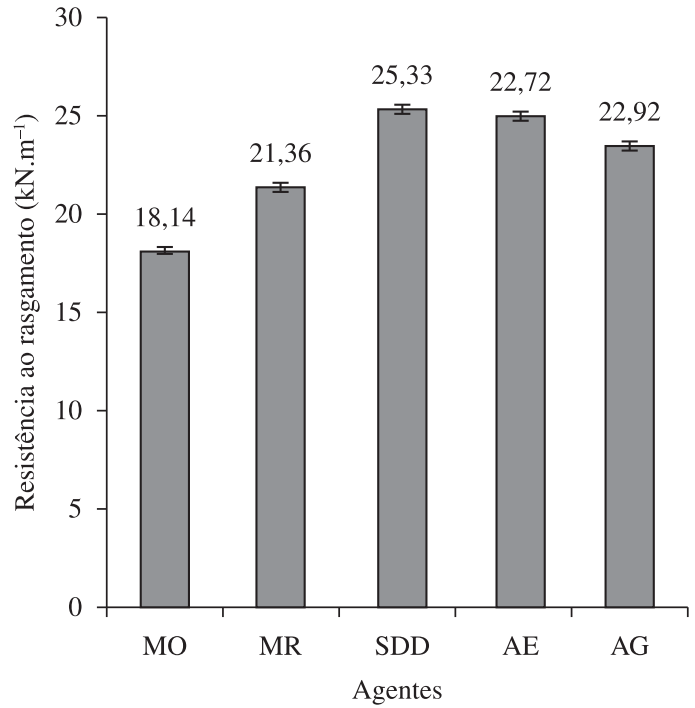

Figura 5. Efeito da adição de agentes na resistência ao rasgamento, erro de $\pm 2 \%$.

Na Figura 1b é interessante observar que a deformação na ruptura diminui acentuadamente com a adição de 80 phr de resíduo (EPDMR), mistura (MR). Embora a carga confira maior resistência mecânica de tração ao material (MR), a deformação dependerá fortemente da distribuição e interação da carga na fase EPDM. Considerando que a carga EPDMR é um material vulcanizado contendo negro de fumo, sua dispersão na fase EPDM é reduzida, favorecendo a formação de agregados de negro de fumo. Essa fase sólida não consegue se deformar e transferir a energia para fase EPDM, causando rompimento prematuro do material, baixa deformação.

A adição de EPDMSDD aumenta acentuadamente à deformação do material (339\%), comparado com as misturas MR (239\%) e MO (275\%), Figura 4b. Este resultado pode ser atribuído ao efeito da estrutura do EPDMSDD na dispersão da carga EPDMR. De uma maneira geral, o resultado é verificado para os agentes EPDMAE e EPDMAG.
Analisando os valores resistência ao rasgamento da amostra (MO) e (MR), Figura 5, nota-se um aumento para composição contendo carga (MR). Observa-se que a resistência ao rasgamento aumenta quando EPDMSDD está presente na mistura, 18\% para SDD. Este resultado sugere que o agente atua diretamente na adesão carga polímero, de modo similar ao que ocorre num sistema de compatibilização ${ }^{[17]}$. O EPDMAE e EPDMAG apresentaram pequenas diferenças em relação à amostra (MR), 6 e 7 \% de aumento, respectivamente. As diferenças encontradas nos resultados podem ser atribuídas à estrutura química dos agentes. O EPDMSDD possui uma cadeia graftizada com 12 átomos de carbormo, enquanto o EPDMAE e o EPDMAG possuem 18 carbonos, sendo que o EPDMAG possui duas duplas ligações, Figura 1. As cadeias com 12 átomos de carbono são reconhecidamente caracterizadas pelo seu efeito saponificante.

\section{Processamento por extrusão}

A Figura 6 mostra o resultado do processamento por extrusão das misturas estudadas, os resultados são apresentados na Tabela 2. A mistura contendo apenas o resíduo (MR) é a que apresenta o valor mais elevado de torque $(38 \mathrm{Nm})$ e valor mais elevado de energia do processamento $(1129 \mathrm{KJ})$. Esse resultado revelou a dificuldade em processar esse material, visto na prática.

Dentre as amostras estudadas a que contém EPDMSDD (SDD) apresentou melhor processamento, valor de torque $(23 \mathrm{Nm})$ e o menor gasto de energia (342 KJ). Este resultado pode ser atribuído ao tamanho da cadeia, 12 átomos de carbono, conforme comentando anteriormente. Além disso, por apresentar uma molécula de enxofre, o EPDMSDD tem um caráter mais ácido quando comparado com os agentes EPDMAG e EPDMAG. O caráter mais ácido contribui para uma melhor dispersão e interação do resíduo com a matriz EPDM. A massa de polímero extrusado é menor quando o EPDMSDD é adicionado, mistura (SDD). Este resultado pode ser atribuído à interação que o EPDMSDD promove na adesão carga/matriz. Os agentes EPDMAG e EPDMAE apresentaram um comportamento similar de torque, embora com valor de energia de processamento inferior ao da mistura (MR). Este resultado pode ser atribuído à estrutura dos agentes. Conforme visto a dupla ligação presente no EPDMAG pouco afeta o processamento da mistura, como também as propriedades mecânicas estudadas. 


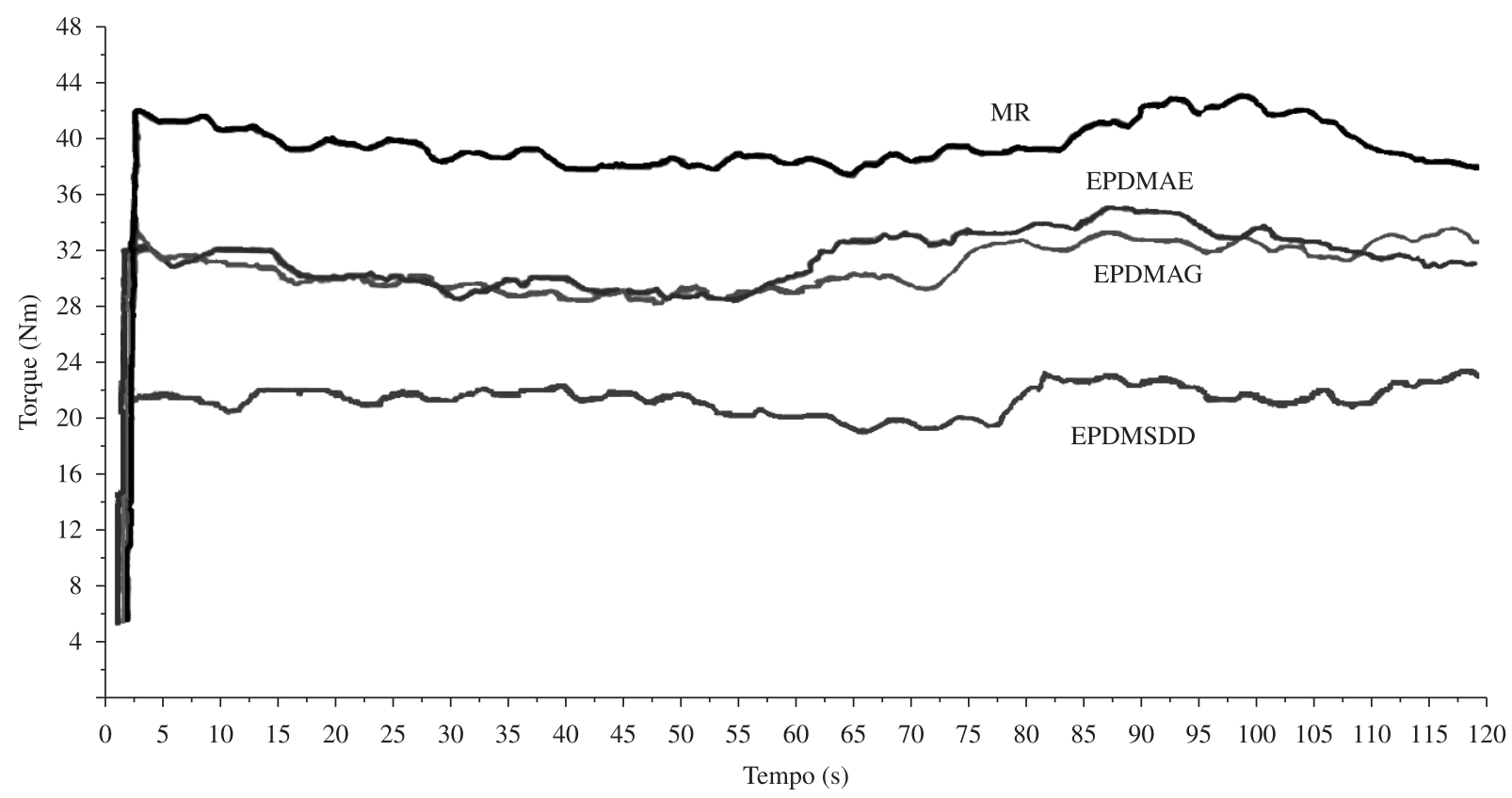

Figura 6. Comportamento do torque em função dos compostos na extrusão em matriz Gravey.

Tabela 2. Dados do processamento por extrusão.

\begin{tabular}{cccc}
\hline Amostras & $\begin{array}{c}\text { Torque } \\
(\mathbf{N m})\end{array}$ & $\begin{array}{c}\text { Energia } \\
(\mathbf{K J})\end{array}$ & $\begin{array}{c}\text { Massa de } \\
\text { extrusado }(\mathbf{g})\end{array}$ \\
\hline EPDMR & 38,0 & 1129 & 18,11 \\
EPDMSDD & 23,0 & 342 & 12,90 \\
EPDMAE & 31,0 & 779 & 16,73 \\
EPDMAG & 28,8 & 692 & 16,45 \\
\hline
\end{tabular}

\section{Conclusões}

O resultado obtido nesse trabalho mostrou ser possível melhorar à recuperação de resíduo de borracha EPDMR, utilizando EPDM modificados com ácidos. Os resultados de propriedades mecânicas mostraram que a presença do agente EPDMSDD resultou nos melhores resultados de resistência a tração e de rasgamento. Os agentes EPDMAE e EPDMAG produziram pouco efeito na resistência à tração e rasgamento. Dentre os agentes estudados o mais efetivo no processamento foi o EPDMSDD, fato este atribuído a sua estrutura de 12 átomos de carbono a qual apresenta um maior efeito saponificante. Os agentes de cadeia longa, EPDMAE e EPDMAG, também produziram melhorias no processamento quando comparado com a amostra MR, indicando sua ação como agente de processamento. Comparando os resultados encontrados para esses dois agentes é possível dizer que à presença de insaturações na cadeia graftizada do EPDMAG pouco afeta as propriedades mecânicas e o processamento.

\section{Agradecimentos}

Os autores desse trabalho agradecem o apoio das seguintes organizações: Decanato de Graduação da UFRRJ, ao PPGEQ, ao INT, a FAPERJ, ao CNPq-PIBIC, a UCS e a Nitriflex S.A.

\section{Referências Bibliográficas}

1. Myhre, M. \& Mackillop, D. A. - Rubber Chem. Technol., 75, p. 429 (2002).
2. Adhikari, B.; De, D. \& Maiti, S. - Prog. Polym. Sci., 25, p 909 (2000).

3. Leblanc, J. L. - Prog. Polym. Sci., 27, p.627 (2002).

4. Payne, A. R. - J. Appl. Polym. Sci., 6, p.57 (1962).

5. Fröhlich, J.; Neideremeyer, W. \& Luginsland, H. - Composities A., 36, p.449 (2005).

6. Ishiaku, U. S.; Chong, C. S. \& Ismail, H. - Polym. Testing., 18, p.621 (1999).

7. Barra, G. M. O.; Roeder, J.; Soldi, V. \& Pires, A. T. N. - Polímeros, 13, p.94 (2003).

8. Pritchard, G. - "An A-Z referenc”, in: Polymer plastics additive, cap.1, Chapman \& Hall, Londres (1998).

9. Baeta, D. A.; Zattera, A. J.; Oliveira, M. G. \& Oliveira, P. J. - Braz. J. Chem. Eng., 26, p.23 (2009).

10. Wang, X. \& Luo, X.- European Polymer Journal., 40, p.2391 (2004).

11. Jacobi, M. M.; Pedrini Neto, C.; Schnieder, C. G.; Rocha T. L. A. C. \& Schuster, R. H. - Kautsch. Gummi. Kunsts., 55, p.590 (2002).

12. Rocha, T. L. A. C. \& Schuster, R. H. - Polímeros., 14, p.318 (2004).

13. Silverstain, R. M.; Bassler, G. C.\& Morril, T. M. - "Identificação espectroscópica de compostos orgânicos", $5^{\circ}$ edição, Guanabara (1994).

14. Carmem, C. J.; Harrington, C. E.\& Wilkers, C. E. - Macromolecules, 10, p.530 (1997).

15. Randall, J. C.- Macromolecules., 11, p.33 (1978).

16. Winters, R.; Lugtenburg, J; Litvinov, V. M.; Duin, M. V. \& Groot, H. J. M. - Polymer, 42, p.9745 (2001).

17. Jansen, P. \& Soares, B. G. - J. Appl. Polym. Sci., 79, p.193 (2001). 